\title{
Investigation of the antioxidant activity of the humic substances and their quinone derivatives before and after reduction by $\mathrm{NaBH}_{4}$
}

Volikov A.B., Perminova I.V.

Lomonosov Moscow State University, Russia, ab.volikov@gmail.com

doi: 10.36291/HIT.2019.volikov.099

Humic substances exhibit antioxidant activity due to phenolic and other groups in their structure. At the same time, some of the groups in their structure are in the oxidized form, for example, quinones, converting them to the reduced form, it is possible to increase the antioxidant activity of humic substances. Based on the difference in antioxidant activity, amount of oxidized groups in the structure can be estimated.

Two humics samples were used in this work: humic substances of leonardite - HS (Humintech, Germany) and fulvic acids of groundwater - FA (Humintech, Germany). To study the more oxidized form of humic substances, quinone derivatives of hydroquinone (HS-HQ, FA-HQ) and pyrocatechol (HA-PQ, FA-PQ) were obtained by Fenton reagent $\left(\mathrm{Fe}^{2+}, \mathrm{H}_{2} \mathrm{O}_{2}\right)$ treatment in alkaline condition. The reduced form of humics was obtained by $\mathrm{NaBH}_{4}$ treatment; for this, $5 \mathrm{ml}$ of a $5 \mathrm{~g} / \mathrm{l}$ solution of $\mathrm{NaBH}_{4}$ was added to $5 \mathrm{ml}$ of a 200 $\mathrm{mg} / \mathrm{l}$ solution of humics sample, kept for 24 hours, after which excess $\mathrm{NaBH}_{4}$ was removed by adding $1 \mathrm{M} \mathrm{HCl}$ to $\mathrm{pH}=7$. Determination of antioxidant activity was carried out using the ABTS radical according to the widely used method, Trolox was used as a standard, the measurement results of Trolox equivalent antioxidant capacity (TEAC) are shown in the figure below.

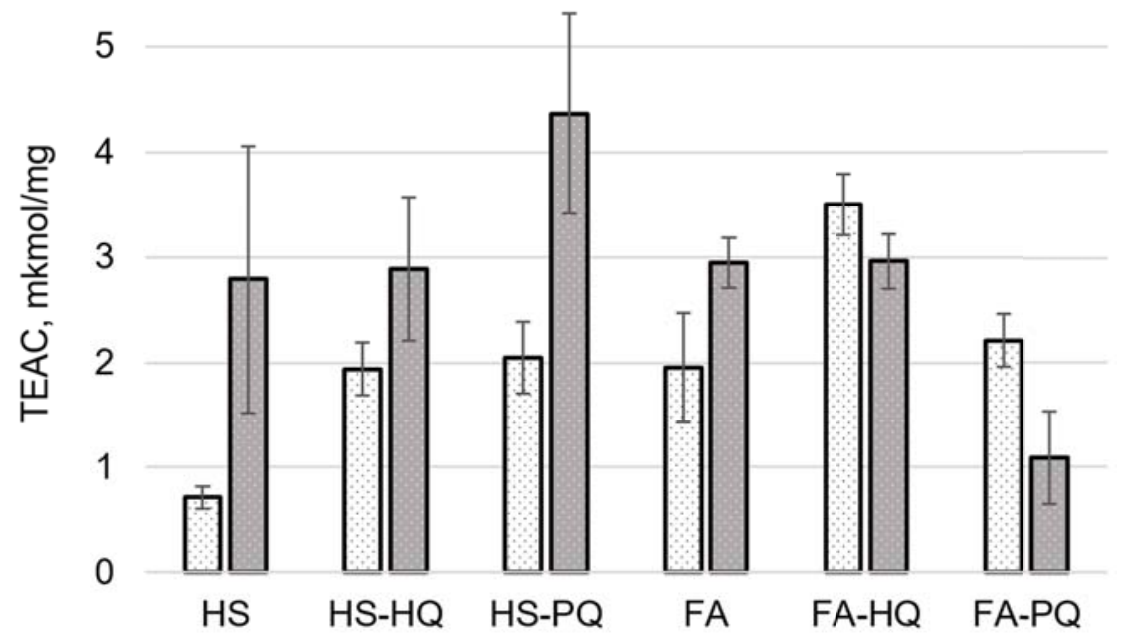

Figure 1. TEAC of humic substances and their quinone derivatives before $\mathrm{NaBH}_{4}$ treatment (white columns) and after (gray columns). ( $P=0.95, n=3)$.

As can be seen from the results, TEAC of HS sample are noticeably lower than FA, which can be explained by the large contribution of phenolic groups to the structure of fulvic acids. But after $\mathrm{NaBH}_{4}$ treatment the value of TEAC is compared amounts to 3 $\mu \mathrm{mol} / \mathrm{mg}$, which is caused by the transition of quinone groups to hydroquinone form in HS. Quinone derivatives show an average TEAC activity - about $2 \mu \mathrm{mol} / \mathrm{mg}$, and their reduction by $\mathrm{NaBH}_{4}$ leads to an increase TEAC in HS derivatives and a decrease in FA derivatives, which may be associated with partial destruction of individual groups in relatively small fulvic acid molecules. If we pay attention to the difference between the initial and reduced forms, the initial sample of humic substances - HS, as well as their pyrocatechol derivatives are of the greatest values, which indicates the greatest contribution of oxidized fragments to their structure.

This research was supported by the RFBR (grant \# 18-29-25065). 